\title{
Technè
}

La science au service de l'histoire de l'art et de la préservation des biens culturels

$45 \mid 2017$

Bronzes grecs et romains : études récentes sur la statuaire antique

\section{Progrès de la recherche sur la statuaire antique en bronze}

Introduction

Advances in the research on ancient bronze statuary

\section{Sophie Descamps-Lequime et Benoît Mille}

\section{(2) OpenEdition}

\section{Journals}

Édition électronique

URL : http://journals.openedition.org/techne/1219

DOI : 10.4000/techne.1219

ISSN : 2534-5168

\section{Éditeur}

C2RMF

Édition imprimée

Date de publication : 1 mai 2017

Pagination : 4-13

ISBN : 978-2-7118-6408-9

ISSN : 1254-7867

\section{Référence électronique}

Sophie Descamps-Lequime et Benoît Mille, " Progrès de la recherche sur la statuaire antique en bronze », Technè [En ligne], 45 | 2017, mis en ligne le 19 décembre 2019, consulté le 22 juillet 2020. URL : http://journals.openedition.org/techne/1219; DOI : https://doi.org/10.4000/techne.1219

\section{(c) (i) $\odot$}

La revue Technè. La science au service de l'histoire de l'art et de la préservation des biens culturels est mise à disposition selon les termes de la Licence Creative Commons Attribution - Pas d'Utilisation Commerciale - Pas de Modification 4.0 International. 


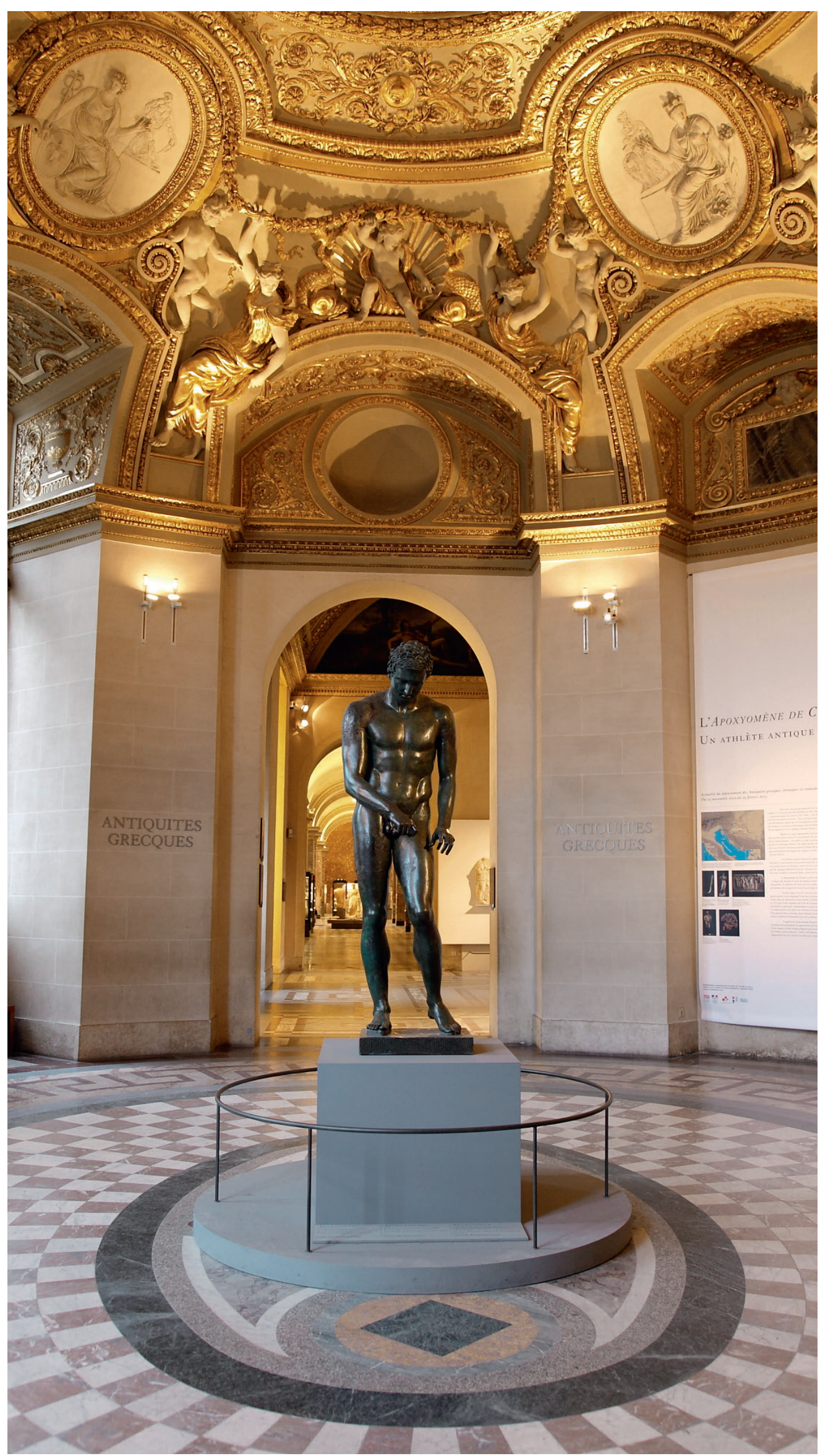

Fig. 1. L’Apoxyomène de Croatie, exposé dans la

Rotonde de Mars du musée du Louvre (23 novembre 2012-23 février 2013). Époque hellénistique. H. 1,92 m. @ Musée du Louvre/A. Mongodin. 
Sophie Descamps-Lequime Benoît Mille

\section{Progrès de la recherche sur la statuaire antique en bronze}

\section{Introduction}

\section{Une journée d'études sur les grands bronzes antiques}

L'exposition au musée du Louvre de l'Apoxyomène de Croatie (fig. 1), repéré en 1996 dans les profondeurs de la mer Adriatique au large de l'île de Lošinj, est à l'origine de l'organisation à Paris d'une journée d'études sur les techniques d'élaboration et la datation des grands bronzes antiques, en février $2013^{1}$. Les actes de cette journée, à laquelle ont participé des spécialistes venus de Grèce, d'Autriche, des États-Unis d'Amérique, de Suisse, de Croatie et de France, sont présentés dans le volume 45 de Technè. Sans l'intérêt immédiat de Brigitte Bourgeois, conservateur général du patrimoine au C2RMF et éditrice de la revue, pour ce domaine de recherche, ils n'auraient pu être publiés. Nous tenons à l'en remercier très sincèrement ${ }^{2}$. Notre reconnaissance va également à Isabelle Pallot-Frossard, directrice du C2RMF, et au comité de rédaction pour avoir accepté la publication de la journée d'études dans la programmation de la revue. Certains intervenants ont présenté des synthèses sur la nature des grands bronzes préservés - originaux isolés, œuvres de série et surmoulages -, sur leurs relations avec les sources littéraires, sur leur production, sur leurs techniques, sur leur circulation, sur les indices objectifs qui fondent leur datation et sur la manière dont l'histoire de la sculpture antique s'est construite au gré des découvertes successives. D’autres ont préféré s'intéresser à des cas particuliers afin de mettre en lumière aussi bien l'existence de multiples que celle de restaurations antiques.

\section{Des répliques antiques en bronze}

La datation des grands bronzes grecs et romains repose en grande partie sur l'étude du contexte archéologique dans lequel ils ont été retrouvés et des moyens techniques mis en œuvre pour les fabriquer. La découverte de l'Apoxyomène
Advances in the research on ancient bronze statuary de Croatie, remonté à la surface en 1999, est venue enrichir le dossier encore très ténu des statues de bronze qui doivent être considérées non comme des créations originales, mais comme des répliques antiques de prototypes disparus. Elle souligne que la démarche qui consistait à nécessairement considérer les quelques bronzes parvenus jusqu'à nous comme des originaux, par opposition aux copies bien plus nombreuses, sculptées dans le marbre et dans d'autres pierres, doit être nuancée : les multiples et les adaptations en bronze existaient aussi. Il est cependant très difficile d'apprécier l'ampleur d'un tel phénomène car ces répliques ont été fondues autant que les œuvres, également en bronze, dont elles déclinaient l'image. Encore faut-il les distinguer et pouvoir les dater. L'analyse stylistique n'est pas suffisante ; les études philologique et épigraphique non plus. Ces approches sont certes incontournables, mais ne peuvent à elles seules permettre d'établir si une statue de bronze est une création originale ou une réplique, et, quand il s'agit de dater l'œuvre en question, de faire la part entre les époques classique, hellénistique et impériale romaine. L'approche technologique et les analyses conduites en laboratoire sont également essentielles. Les conclusions les plus plausibles ne peuvent naître que de discussions collégiales entre historiens et historiens d'art, épigraphistes, physico-chimistes, radiologues et restaurateurs, fondées sur la mise en commun des connaissances et des interprétations de chacun.

\section{Des œuvres rarement conservées}

Les grands bronzes antiques, tant grecs que romains, sont extrêmement rares. On sait, grâce aux sources textuelles et aux indices archéologiques matériels, que des milliers de statues de bronze ornaient les sanctuaires comme les places publiques, les palais et les demeures de l'élite à partir de l'époque hellénistique, les thermes et les jardins romains. Mais, dans leur très grande majorité, ces œuvres n'ont pas

Sophie Descamps-Lequime, conservateur général du patrimoine, musée du Louvre, département des Antiquités grecques, étrusques et romaines (sophie.descamps@louvre.fr). Benoît Mille, ingénieur d'étude, C2RMF et Préhistoire et Technologie, UMR 7055, Nanterre (benoit.mille@culture.gouv.fr). 
survécu à l'Antiquité car elles ont été refondues pour recycler leur alliage. Les bronzes préservés ont échappé fortuitement à la refonte car ils n'étaient déjà plus visibles à la fin de l'Antiquité, soit parce qu'ils appartenaient à la cargaison de bateaux naufragés ou étaient des ex-voto endommagés et ensevelis sur place, soit parce qu'ils se trouvaient dans des constructions effondrées lors d'incendies ou de tremblements de terre. C'est la raison pour laquelle toute nouvelle mise au jour d'un grand bronze est saluée par la communauté scientifique comme un événement majeur pour l'histoire de la sculpture antique. Étant «brut de fouille » et avec la performance actuelle des examens disponibles, ce bronze peut bénéficier d'une étude fondamentale, durant et même après sa restauration. Les questions qui se posent concernent auteur ou à une école, sa date et sa qualité d'original ou de réplique antique. S'il est possible de répondre à l'une ou à plusieurs de ces questions, l'œuvre nouvellement introduite dans le petit corpus de la statuaire antique en bronze devient un jalon auquel confronter d'autres sculptures, parfois connues depuis quelques décennies, voire plusieurs siècles, mais qui, parce qu'elles ont pu subir plusieurs interventions modernes, livrent un message plus difficile à décrypter.

\section{L'Apoxyomène de Croatie : une œuvre hellénistique}

L'Apoxyomène de Croatie $^{3}$ témoigne de la complexité des dossiers et de la nécessité de définir des critères objectifs afin de renforcer l'apport des données historiques et stylistiques, par l'association d'un cadre fondé sur une évolution technologique, à la fois diastratique et diachronique (fig. 1). La présence de l'Apoxyomène à Paris a conduit le C2RMF à compléter le dossier documentaire par une nouvelle couverture radiographique exhaustive de la statue (face et profil) et à effectuer des analyses de composition élémentaire par ICP-AES des différents alliages employés. Ces examens et analyses ont permis de mieux identifier les matériaux constitutifs, et d'aboutir à une meilleure compréhension des techniques de fonte, d'assemblage et de décor de la statue ${ }^{4}$ (voir aussi base Héphaïstos ci-après).

Retrouvé avec une plinthe non décorée sur la face postérieure, qui indique qu'il ornait, dans un second temps au moins, une niche ou était disposé le long d'un mur et était destiné par conséquent au décor d'une villa, de bâtiments publics - thermes ou gymnase - ou d'un jardin, l'Apoxyomène de Croatie renvoie stylistiquement au IV ${ }^{\mathrm{e}}$ siècle av. J.-C. Mais plusieurs détails techniques - la soudure en plateforme entre la tête et le cou ou la forme polygonale et les dimensions de certaines pièces de réparure qui dissimulent les défauts de la fonte après la coulée - imposent de descendre sa date et de le considérer non comme la création même de l'artiste, mais comme une réplique antique, un multiple de l'œuvre originelle disparue. Par ailleurs, la nouvelle campagne d'analyses par ICP-AES conduite au C2RMF a permis d'éclairer d'un jour nouveau la composition élémentaire de la statue :

les résultats montrent définitivement que l'Apoxyomène de Croatie a été fabriqué à partir d'un bronze à fort taux de plomb $(6,7 \pm 1,9 \% \text { d'étain, } 18,0 \pm 4,0 \% \text { de plomb })^{5}$. Or cette composition n'est pas compatible avec une datation au IV siècle av. J.-C. Lors du séjour de la statue au C2RMF, une attention toute particulière a été portée aux techniques de fabrication de la bouche. La nouvelle radiographie du profil de la tête a permis de vérifier que les lèvres en cuivre rouge non allié ne sont pas massives, comme on l'aurait attendu de la statue si elle avait été du IV ${ }^{\mathrm{e}}$ siècle av. J.-C., tel l'Éphèbe d'Anticythère (Athènes, Musée archéologique national) ${ }^{6}$. Elles sont incrustées à froid, indice d'une production postérieure ${ }^{7}$. Après la coulée, deux sillons de $4 \mathrm{~mm}$ de profondeur et de hauteur ont été incisés dans le bronze, tout le long des lèvres supérieure et inférieure. Les inserts en cuivre ont été incrustés dans ces sillons, puis martelés de façon à appliquer une feuille de cuivre d'environ $1 \mathrm{~mm}$ d'épaisseur qui recouvre l'intégralité de la surface des lèvres.

L'absence de contexte empêche de savoir quand la statue a été transportée. On ne peut déterminer précisément non plus quelle était sa destination et quelle était sa provenance. Mais des analyses au ${ }^{14} \mathrm{C}$ ont permis de dater certains épisodes subis par l'œuvre, parmi lesquels une ou deux phases de restauration entre la seconde moitié $\mathrm{du} \mathrm{I}^{\mathrm{er}}$ siècle et le $\mathrm{II}^{\mathrm{e}}$ siècle de notre ère. L'une de ces restaurations a été confirmée par les nouvelles analyses du métal, puisqu'il s'avère que la face postérieure non décorée de la plinthe est de même composition que le reste de la statue, tandis que les côtés et la partie antérieure, qui portent un décor, sont d'un alliage et de spectre d'impuretés différents de tout le reste, ce qui suggère une intervention majeure sur l'Apoxyomène de Croatie à une époque postérieure à la fabrication de la statue, mais toutefois durant le cours de sa «vie » antique. Enfin, les vestiges retrouvés à l'intérieur de la statue et leurs analyses ${ }^{14} \mathrm{C}$ indiquent une possible période d'abandon, durant laquelle un petit rongeur a pu amasser de la nourriture dans la jambe droite et faire son nid dans l'avant-bras gauche. L'analyse au ${ }^{14} \mathrm{C}$ d'un noyau de pêche mordu par le rongeur a conduit à dater le moment où l'athlète de bronze devait être couché sur le sol entre 110 av. J.-C. et 70 apr. J.-C., avec une forte probabilité vers 20 av. J.-C., donc avant la fin du I ${ }^{\text {er }}$ siècle av. J.-C. Tous les résultats convergent donc vers une information de la plus grande importance : tous les bronzes connus à ce jour ne sont pas nécessairement les œuvres d'origine mais peuvent être des répliques antiques des prototypes disparus et par ailleurs, il existait bien des surmoulages de bronze dès l'époque hellénistique ${ }^{8}$.

\section{La base de données Héphaïstos}

Lors de la journée d'études de 2013, nous avions évoqué le principe d'une base de données conçue comme un outil d'étude des techniques de fabrication des grands bronzes antiques, destiné à être partagé par la communauté scientifique concernée. Fondée sur un partenariat entre le Louvre et le 
C2RMF, elle est en cours de construction. L'étude d'un bronze antique impose, nous l'avons vu avec l'Apoxyomène de Croatie, de comprendre d'abord comment il a été fabriqué. L'analyse technologique est plus importante sans doute que dans d'autres domaines de la création artistique antique. Les spécialistes des bronzes le savent : sans cette approche totalement indépendante des autres, les risques d'erreurs sont majeurs. La base de données Héphaïstos est donc née de ce constat selon lequel on ne peut, pour une œuvre de bronze, aborder les questions de style et de datation sans avoir également rassemblé tous les indices et toutes les données matérielles qui permettent de suivre son élaboration depuis le modèle originel conçu par l'artiste. C'est dans ce sens que travaillent désormais tous les spécialistes des grands bronzes antiques, engagés dans cette voie par les premières études d'Edilberto Formigli sur les deux guerriers de Riace ${ }^{9}$. Les publications d'œuvres en bronze se présentent souvent comme des monographies très complètes mais indépendantes les unes des autres. La base de données Héphaïstos est en conséquence fondée sur le dépouillement le plus large possible des ouvrages ou articles qui traitent des statues de bronze. Soulignons ici que les dimensions importent assez peu car de très petits bronzes peuvent présenter parfois les mêmes caractéristiques techniques que la grande statuaire. Un critère simple a été retenu pour la sélection des bronzes, à savoir l'emploi du procédé de fonte en creux : l'œuvre peut donc aussi bien être une statuette.

La base de données repose sur l'établissement d'un système descriptif précis. Son objectif est de permettre l'étude de la variation spatio-temporelle des techniques de fabrication des grands bronzes antiques. La fiche Héphä̈stos de l'Apoxyomène de Croatie illustre ici la base de données (fig. 2). Les fiches d'œuvres rassemblent dans une première partie les informations d'ordre archéologique (contexte de découverte), iconographique et stylistique (attribution, atelier) et la datation tirée de ces données. La précision et la fiabilité de la datation de l'œuvre sont des questions primordiales dans le contexte de la base Héphaïstos, puisque la portée des informations technologiques révélées par l'étude d'une statue particulière en dépend entièrement. Pour éviter l'écueil d'un raisonnement circulaire, elle s'appuie au maximum sur les données externes, et non sur la technique ${ }^{10}$. Dans une deuxième partie, tous les indices technologiques relevés sur la statue sont précisément et systématiquement décrits : nombre de pièces coulées séparément, épaisseur moyenne des parois, type de procédé de fonte à la cire perdue, traces du travail de la cire et du noyau, nature du dispositif de maintien du noyau, procédés d'assemblage, décor, réparure. Dans une dernière partie, la composition élémentaire de l'alliage est indiquée, ainsi que la liste des examens et analyses effectués en laboratoire. Deux tables liées complètent chaque fiche. La première rassemble l'intégralité des analyses élémentaires (report des résultats multiples lorsque plusieurs analyses ont été effectuées sur une statue, et transcription des éléments chimiques en impuretés et pas uniquement la composition de l'alliage). La seconde table rassemble les notices bibliographiques ayant permis de constituer chaque fiche Héphaïstos. Enfin, une sélection d'illustrations (neuf au maximum) accompagne chaque ouvre (fig. 3).

La fiabilité de la base relève de la relecture des fiches à la fois par des historiens d'art et des spécialistes des techniques de la grande statuaire. Les données peuvent ensuite être exploitées pour déterminer quelle a pu être l'évolution des techniques de fabrication de la grande statuaire de bronze dans l'espace et dans le temps, et offrir à terme la possibilité d'une datation technologique des bronzes ${ }^{11}$, ainsi que des éléments qui permettront de conforter l'hypothèse d'un lieu de production ${ }^{12}$ (fig. 4).

Dans son état actuel, la base de données Héphaïstos implique donc un important effort de validation par la communauté scientifique. Certaines exploitations sont néanmoins d'ores et déjà possibles. À ce stade, la base compte 775 entrées et la proportion des œuvres analysées est non négligeable : la table « analyses élémentaires du métal » compte ainsi près d'un millier de résultats. Au prix d'un tri drastique, il est possible de conserver 165 grandes statues correctement datées comme références de composition élémentaire, ce qui permet de décrire pour la première fois sur près de 1000 ans l'évolution de la composition élémentaire des alliages de la grande statuaire antique (fig. 5). On constate ainsi que la composition des alliages était très soigneusement contrôlée : on ne connaît aucun grand bronze antique constitué à partir d'un alliage cuivre-zinc (laiton), alors que cet alliage est d'un emploi extrêmement fréquent pour la petite statuaire d'époque romaine et gallo-romaine. Cette volonté d'éviter délibérément le zinc suggère une motivation d'ordre technique (problèmes pendant le soudage en présence de zinc ?). On observe aussi que la teneur en étain diminue avec le temps. Cependant, le phénomène est extrêmement lent, et la dispersion est très importante. La teneur en étain ne peut donc pas être utilisée pour discuter de la chronologie d'une seule et unique statue. Enfin, le plomb ne dépasse jamais $5 \%$ massique avant l'avènement de la période hellénistique ( $\mathrm{III}^{\mathrm{e}} \mathrm{s}$. av. J.-C.). Pendant les époques hellénistique et romaine, les bronzes à faible, moyenne ou haute teneurs en plomb ont en revanche été utilisés sans distinction. Si la composition des alliages peut donc contribuer à la discussion sur la datation, elle ne sera probablement jamais un argument suffisant. D'autres critères, davantage liés aux techniques de coulée ou d'assemblage, semblent déjà constituer des marqueurs technologiques plus discriminants.

La base Héphaïstos s'est également révélée être un outil de comparaison particulièrement performant. L'un des organisateurs a récemment eu l'opportunité d'étudier les techniques de fabrication de la grande statuaire de bronze des royaumes sabéens (Yémen actuel), qui se trouve être contemporaine de celle des grands bronzes grecs et romains ${ }^{13}$. En première approche, les deux méthodes de fabrication partagent nombre de principes communs, dont en particulier les deux techniques les plus fondamentales : le procédé indirect de fonte à la cire perdue pour couler les statues en pièces séparées, et le soudage par fusion au bronze liquide pour 


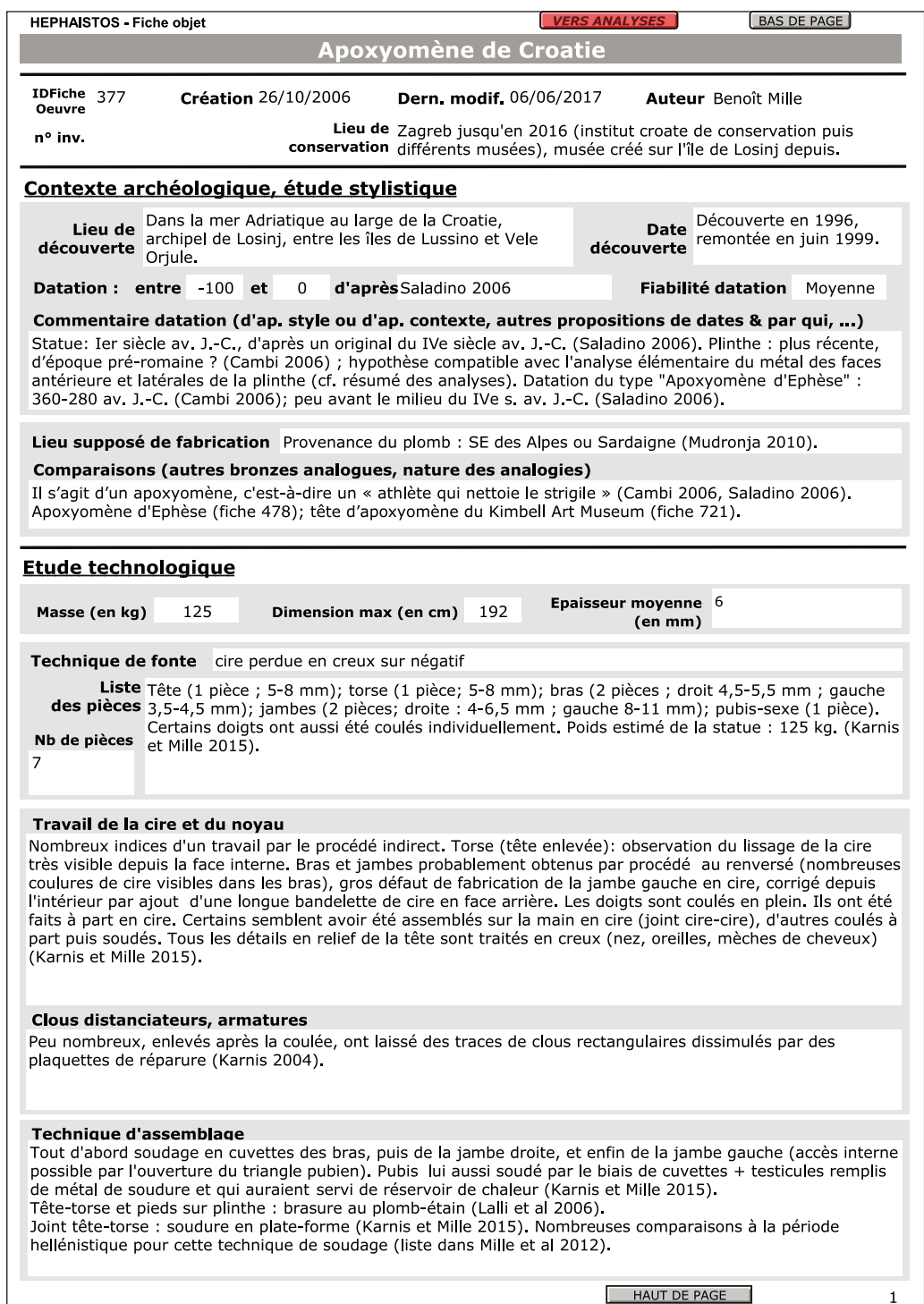

Fig. 2. Exemple d'une fiche d'œuvre extraite de la base de données Héphaïstos : l'Apoxyomène de Croatie.

\section{HEPHAISTOS - Fiche objet \\ Etude technologique (suite) \\ UERS ANALYSES BAS DE PAGE}

Décor Mamelons et lèvres incrustés par des inserts en cuivre non allié (Karnis et Mille 2015), et non lèvres coulées à part en plein et placées dans la cire (Saladino 2006)

Plinthe métallique qui recouvrait le sommet étroit d'une base en pierre rectangulaire, constituée de 5 plaques de bronze: plaque supérieuret et bord avant

Yeux Rapportés, perdus (Karnis 2004)

Réparure, ajustage, finitions

Très nombreux défauts liés aux dégagements gazeux pendant la coulée (bulles), aux épaisseurs irrégulières (notamment sous-épaisseur jambe droite porteuse) et aux assemblages soudés. Plusieurs centaines de réparures ont été nécessaires pour cacher ces défauts : petites plaquettes rectangulaires, grandes plaquettes polygonales, coulées secondaires pour combler les lacunes les plus importantes (Karnis 2004, Karnis et Mille
Aissell Aisselle droite : renfort en bois analysé au $14 \mathrm{C}$ (restauration antique ?) : daté entre 30 av. et 130 ap. J.-C. 50-170 ap. J.-C., date la plus probable v. 110 ap. J.-C. (Saladino 2006).

\section{Remarques}

Matériaux organiques à l'intérieur de la statue. Analyses paléobotaniques et archézoologiques : brindilles de bois, feuilles de laurier, noyaux, fruits et restes d'insectes. Nid d'un petit rongeur composé de foin de feuilles au 14C (Beta Analytic), entre 20 av. J.-C. et 110 ap. J.-C., milieu : la Méditerranée (L'atleta della Croazia 2006).

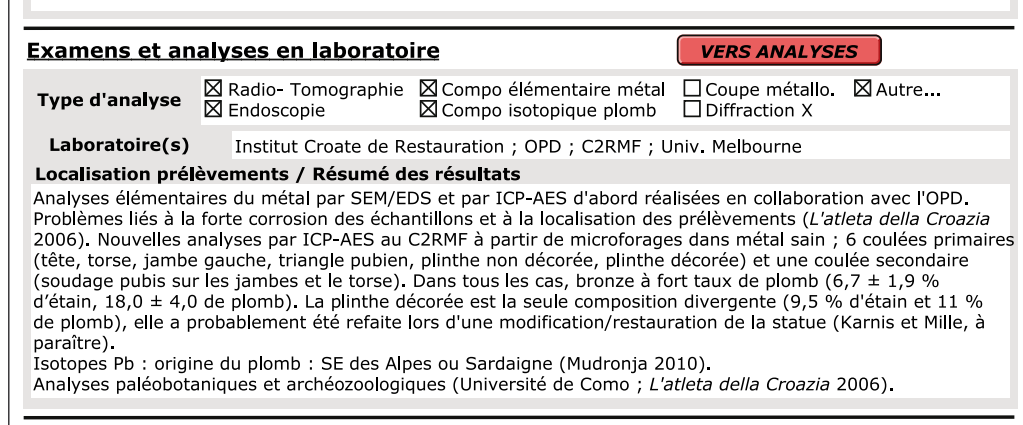

\section{Bibliographie}

ID Fiche biblio

705

706

707

708

709

710

1528

1529

1530
Référence bibliographique abrégée

Mudronja 2010

Cambi 2006

Karnis 2004

L'atleta della Croazia 2006

Saladino 2006

Lalli et al 2006

Karnis et Mille 2015

Karnis et Mille 2017

Mille et al 2012

\section{Accès fiche $\square$




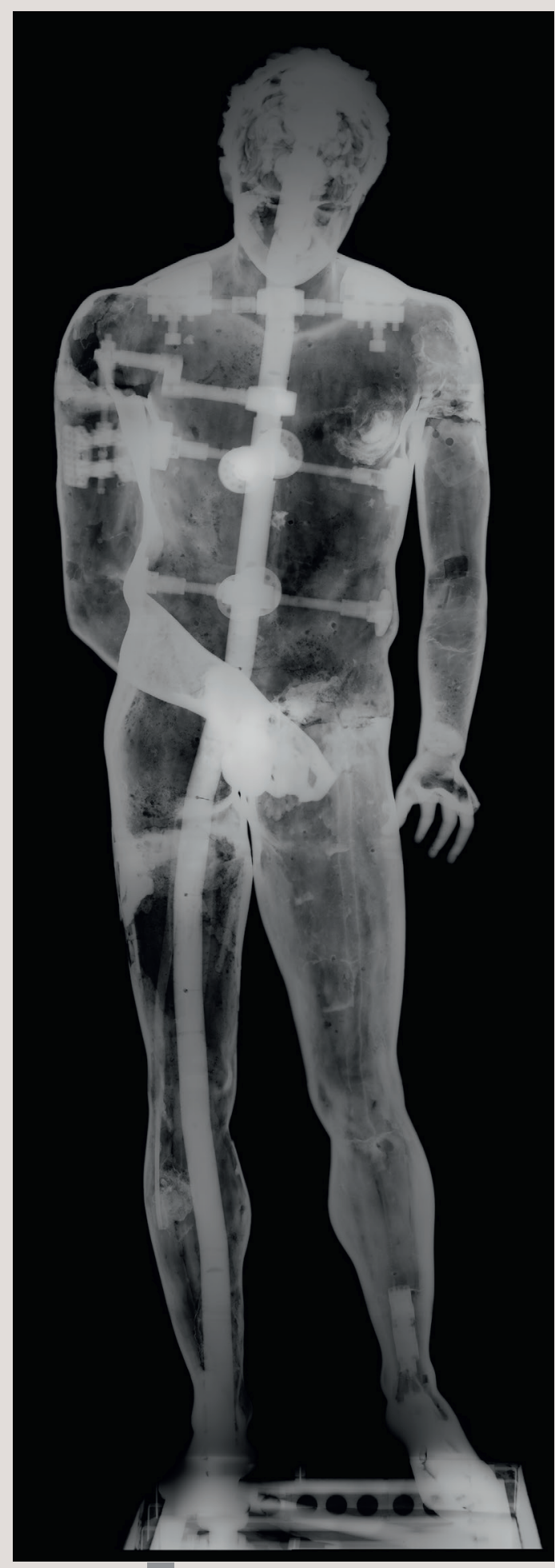

a

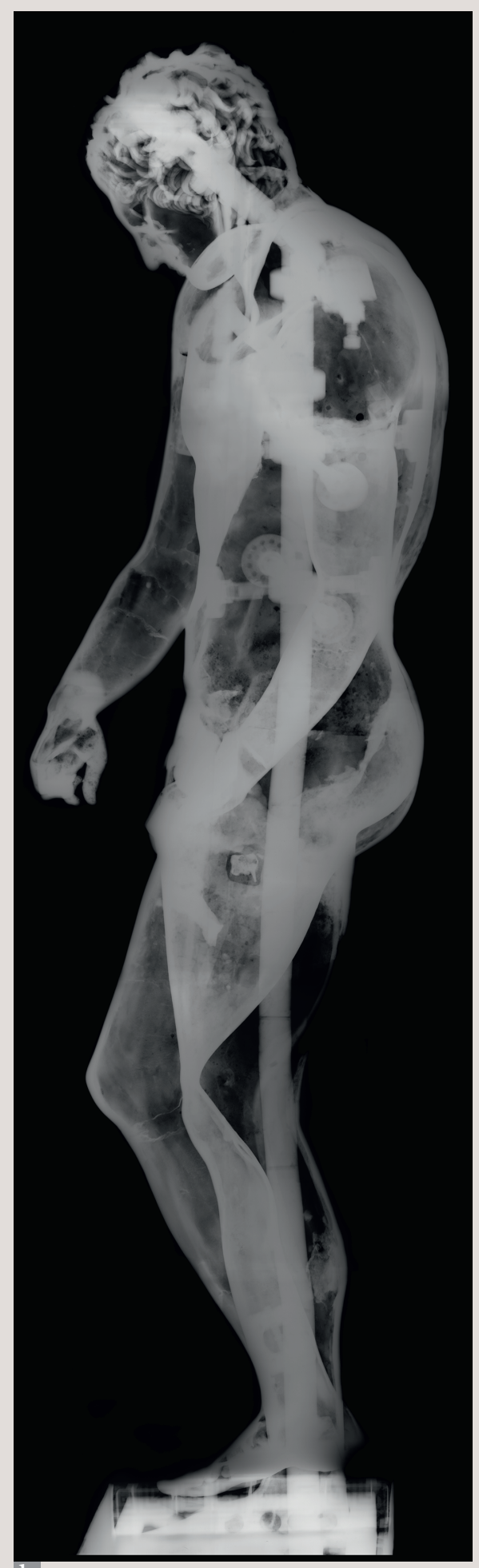

$\mathrm{b}$
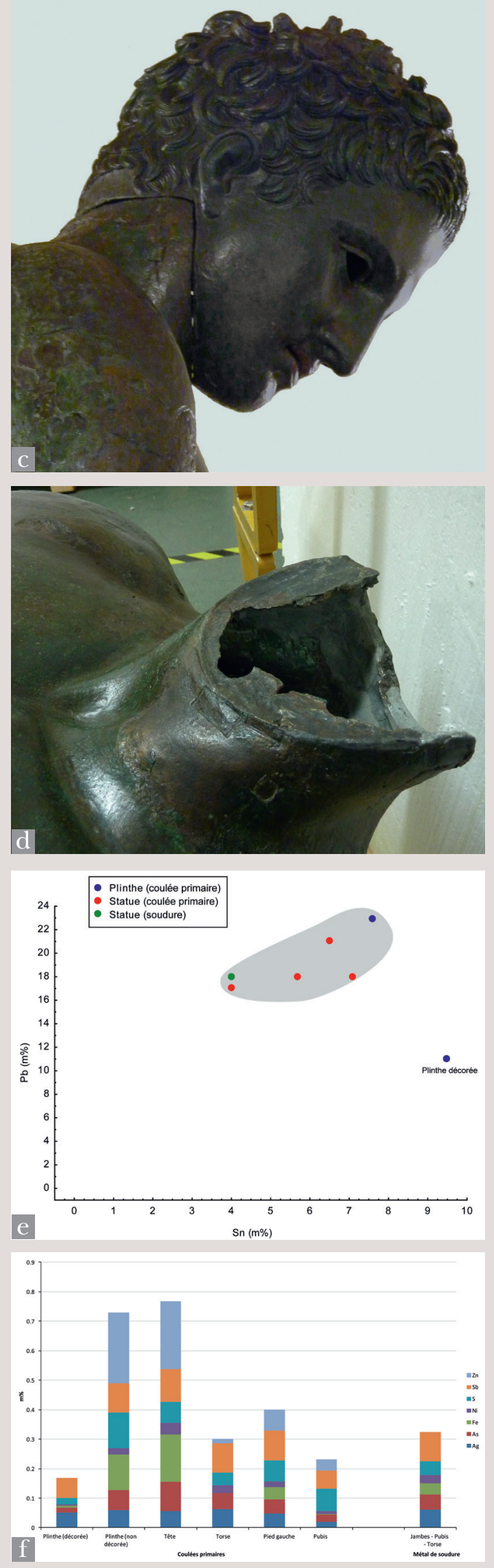

Fig. 3. Illustrations accompagnant la fiche Héphaïstos de l'Apoxyomène de Croatie. (a) radiographie $\mathrm{X}$, vue de face; (b) radiographie X, vue de profil ; (c) soudure en plateforme de la tête sur le torse, vue de profil, (d) détail de la plateforme préparée pour le soudage côté torse, tête démontée ; (e) composition élémentaire du métal, diagramme binaire étain-plomb ; (f) composition élémentaire du métal, teneurs cumulées des principales impuretés. (a) et (b) : ( ) C2RMF/E. Lambert et J. Marsac ; (c) à (f) : @ C2RMF/B. Mille. 
assembler ces pièces. Les caractéristiques des statues sabéennes se prêtent sans difficulté à un enregistrement dans la base Héphaïstos, et nous avons ainsi renseigné une cinquantaine d'œuvres conservées dans les collections des musées du Yémen. Cela a ensuite permis de confronter dans le détail les techniques grecques et romaines aux techniques sudarabiques (tableau 1). Il a ainsi été montré que si les statues sudarabiques partageaient les deux principes qui forment la base de la fabrication des grandes statues grecques et romaines, tout ou presque différenciait les deux méthodes dans le détail de la mise en œuvre de ces techniques. Cela suggère un héritage technologique commun très ancien (Proche-Orient, Égypte?), puis une évolution distincte de cet artisanat très spécialisé dans les deux grandes aires culturelles en question.
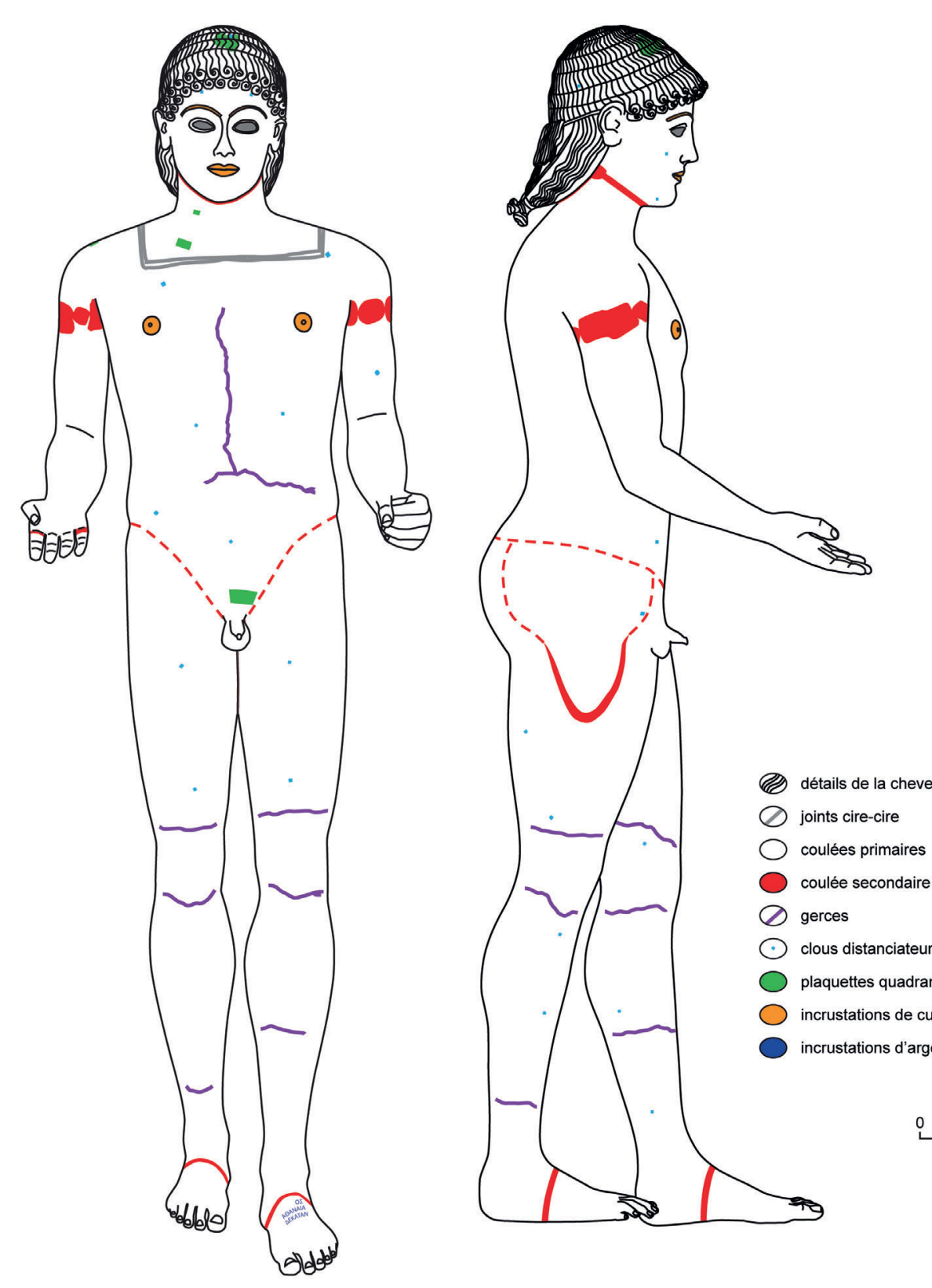

Fig. 4. Relevé de synthèse des observations technologiques effectuées sur l'Apollon de Piombino. Les techniques mises en évidence écartent définitivement l'hypothèse d'une datation des $\mathrm{VI}^{\mathrm{e}} \mathrm{ou} \mathrm{V} \mathrm{V}^{\mathrm{e}}$ siècles au profit de la période hellénistique (notamment l'alliage à fort taux de plomb et le type de soudure de la tête). Par ailleurs, le travail en fonderie a conduit à la production d'une ouvre presque exempte de défauts.

Cette maîtrise tout à fait exceptionnelle pourrait être en relation avec l'origine rhodienne de la statue. @ C CRMF/B. Mille. 

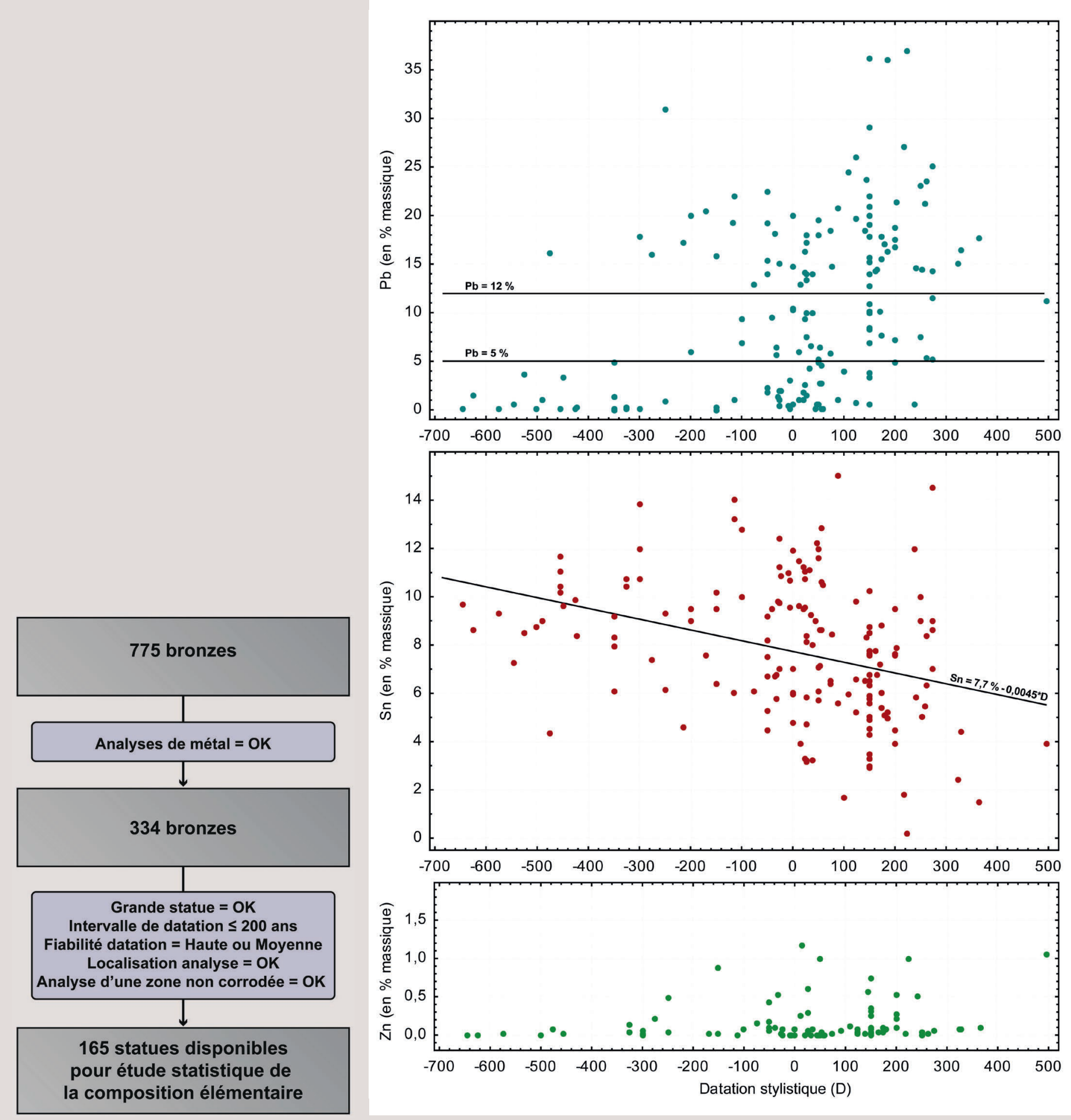

Fig. 5. Évolution de la composition des alliages de la grande statuaire grecque et romaine. @ C 2 RMF/B. Mille. (a) critères de sélection utilisés pour isoler une série d'analyses de référence à partir de la base de données Héphaïstos. (b) Évolution des teneurs en zinc, étain et plomb d'après les analyses de bronzes grecs et romains durant la période 700 av. J.-C.- 500 ap. J.-C. 
Tableau 1. Comparaison des méthodes de fabrication sudarabique/gréco-romaine pour la grande statuaire antique en bronze. La comparaison se fait sur la base de statues coulées en pièces séparées (coulées primaires), ensuite assemblées par un procédé de soudage par fusion au bronze liquide, d'après Mille, 2012.

\begin{tabular}{|l|l|}
\hline Monde sabéen & Monde grec puis romain \\
\hline Premières grandes statues de bronze : vers 600 av. J.-C. & Premières grandes statues de bronze : vers 550 av. J.-C. \\
\hline Procédé indirect de fonte en creux à la cire perdue & Procédé indirect de fonte en creux à la cire perdue \\
\hline Soudage par fusion au bronze liquide & Soudage par fusion au bronze liquide \\
\hline $\begin{array}{l}\text { Fréquent habillage du modèle en cire par des détails sculptés } \\
\text { sur positif tout au long de la période de production }\end{array}$ & $\begin{array}{l}\text { Profusion de détails ajoutés par un travail de la cire sur positif } \\
\text { à la période du style sévère ; beaucoup moins fréquent ensuite }\end{array}$ \\
\hline Les défauts de coulée sont rares & Les défauts de coulée sont très fréquents \\
\hline $\begin{array}{l}\text { Bronze à fort taux de plomb pendant toute la période } \\
\text { de production (-600 à }+300)\end{array}$ & $\begin{array}{l}\text { Bronze binaire, puis bronze à fort taux de plomb (après -300), } \\
\text { usage non systématique }\end{array}$ \\
\hline $\begin{array}{l}\text { Parois métalliques très fines (1-3 mm), depuis -600 jusque } \\
+300\end{array}$ & $\begin{array}{l}\text { Parois métalliques assez épaisses (6 à 12 mm), diminuent } \\
\text { fortement après -300 (2 à 4 mm) }\end{array}$ \\
\hline $\begin{array}{l}\text { Noyau creux aussi souvent que possible, systématiquement } \\
\text { laissé dans la statue }\end{array}$ & $\begin{array}{l}\text { Noyau plein. Retiré au moins partiellement pour le soudage, } \\
\text { toujours enlevé après -300 }\end{array}$ \\
\hline $\begin{array}{l}\text { Préparation du soudage complexe nécessitant de réserver } \\
\text { des espaces à l'étape cire (anneaux), puis de mettre en place } \\
\text { un noyau intermédiaire en plâtre, juste avant le soudage }\end{array}$ & $\begin{array}{l}\text { Simple ajustage mécanique des bords à joindre (cordon linéaire), } \\
\text { éventuellement complété par la découpe de demi-ovales } \\
\text { en vis-à-vis (cuvettes) }\end{array}$ \\
\hline $\begin{array}{l}\text { Soudures en forme d'anneaux (période archaïque), } \\
\text { puis en cordons linéaires }\end{array}$ & $\begin{array}{l}\text { Diverses formes, dont soudure en cordons ponctués de cuvettes / } \\
\text { soudure en plateforme pour la tête et les jambes à partir } \\
\text { de la période hellénistique }\end{array}$ \\
\hline $\begin{array}{l}\text { Composition élémentaire du métal d'apport pour le soudage } \\
\neq \text { Composition élémentaire du métal des coulées primaires }\end{array}$ & $\begin{array}{l}\text { Composition élémentaire du métal d'apport pour le soudage } \\
\text { = Composition élémentaire du métal des coulées primaires }\end{array}$ \\
\hline
\end{tabular}

\section{De l'importance des techniques de soudage}

Dès l'établissement du programme commun de recherche entre le département des Antiquités grecques, étrusques et romaines du Louvre et le C2RMF sur les techniques de la grande statuaire métallique antique, nous avons souligné que les techniques de soudage jouent un rôle essentiel dans la fabrication des grands bronzes grecs et romains ${ }^{14}$. Entre 2010 et 2013, une thèse de doctorat de l'université Paris VI a permis des avancées considérables dans la compréhension des paramètres thermiques et chimiques associées à ces complexes opérations d'assemblage ${ }^{15}$; la journée d'études a été l'occasion d'exposer l'état d'avancement de ces recherches.

Par ailleurs, toujours dans le cadre de ce programme, une collaboration a été nouée avec l'Institut de Soudure : ont ainsi été produites les premières cartographies des zones soudées d'une statue antique par le biais de techniques ultrasonores très innovantes ${ }^{16}$. Ces techniques, en particulier la possibilité d'aboutir à de véritables tomographies ultrasonores, sont porteuses d'un grand avenir dans le domaine de la science des matériaux du patrimoine ; elles ont également été présentées par l'Institut de Soudure lors de la journée d'études. Soulignons enfin que l'Institut de Soudure s'est beaucoup investi dans l'organisation de cette journée, notamment en la personne de Daniel Chauveau, directeur de l'innovation ; ces quelques lignes sont la marque de notre reconnaissance pour cet important soutien.

\section{Des études complémentaires}

Plusieurs dossiers inédits, sur des bronzes gallo-romains conservés au musée du Louvre, ont été ajoutés aux actes de la journée d'études. Ils illustrent d'autres directions de recherche empruntées par le C2RMF : l'étude de la fabrication des yeux de deux petits bustes d'Auguste et de Livie, exhumés à Neuilly-le-Réal en 1816, a mis en évidence l'existence d'une technique insoupçonnée jusqu'alors, qui associait production verrière de luxe et travail du bronze ; celle de l'Apollon de Lillebonne, découvert en 1823, a apporté des éléments nouveaux sur les techniques de dorure des statues d'époque impériale romaine et confirmé que l'effigie divine a connu, quelques décennies seulement après son érection, une phase de redorure partielle parce qu'elle se dressait en plein air.

Il a été demandé enfin aux spécialistes des surfaces métalliques patinées intentionnellement dans l'Antiquité de faire le point sur la question des «bronzes et cuivres noirs » ou «bronzes de Corinthe " plus de vingt ans après les premières études. Si l'on sait désormais que les patines noires à base de cuprite renferment toujours une faible quantité d'or et/ou d'argent ajoutée volontairement, comment explique-t-on aujourd'hui, à la lumière des progrès réalisés sur les nanoparticules, le phénomène physique étrange, découvert empiriquement par les Anciens, qui conduit l'œil à voir une patine de couleur noire, alors que la cuprite est rouge? 
Notes

1. L’Apoxyomène a été exposé au département des Antiquités grecques, étrusques et romaines du musée du Louvre, du 23 novembre 2012 au 23 février 2013. La journée d'études "Originaux, répliques et pastiches : techniques d'élaboration et datation des grands bronzes antiques " a été organisée par S. Descamps et B. Mille, avec le soutien du musée du Louvre, du C2RMF et de l'Institut de Soudure. Elle a eu lieu le 12 février 2013

2. Nous souhaitons également exprimer notre gratitude à Anne Terral, qui a assuré auprès de Brigitte Bourgeois l'assistance éditoriale de ce numéro de la revue Technè.

3. Voir en dernier lieu Daehner, 2015, avec la bibliographie antérieure.

4. Karniš Vidovič, Mille, 2015 ; Karniš Vidovič, Mille à paraître.

5. Karniš Vidovič, Mille à paraître.

6. Voir dans ce volume, la fig. 6 de l'article de Bouyia, p. 29

7. Mentionnons toutefois les lèvres plaquées de l'Apollon Chatsworth, vers 460-450 av. J.-C., une « anomalie » justifiée par l'attribution de l'œuvre à un artiste chypriote (Bouquillon et al., 2006. p. 255-256)

8. Voir aussi dans ce volume les articles de Mattusch et Plattner.

9. Formigli, 1984. Mentionnons par exemple, à la suite de cette publication fondatrice, celles de Mattusch, 1996 ; Hemingway, 2004 ; Bouquillon et al., 2006 ; Schalles, Pelz, 2011 ; Mille et al., 2012.

10. La date est donnée sous la forme d'un intervalle (par exemple pour l'Apoxyomène de Croatie, entre -100 et 0 ). Nous attribuons aussi un critère de fiabilité haute (datation absolue de la fabrication ; inscription épigraphique, iconographie ou style permettant un ancrage chronologique sans aucune ambiguïté possible) ; moyenne (données ne permettant pas de dater très précisément l'œuvre) ; basse (œuvre dont la datation est très discutée et/ou particulièrement difficile)

11. Formigli, 2012

12. Sur ces questions de datation technologique et de lieu de production, voir aussi notre étude de l'Apollon de Piombino, Mille et Descamps-Lequime, à paraître, et l'article de N. Badoud dans ce volume.

13. Mille, 2012.

14. Bouquillon et al., 2006, p. 243-250.

15. Azéma, 2013.

16. Azéma et al., 2012.

\section{Bibliographie}

Azéma A., Angelini F., Mille B., Framezelle G., Chauveau D., 2012, « Le Gaulois captif d'Arles, étudié au C2RMF et analysé par l'IS grâce aux ultrasons ", Soudage et techniques connexes, 66(9-10), p. $10-15$.

Azéma A., 2013, Les techniques de soudage de la grande statuaire antique en bronze: étude des paramètres thermiques et chimiques contrôlant le soudage par fusion au bronze liquide, Thèse de doctorat de l'université Pierre-et-Marie-Curie (Paris VI), spécialité chimie physique et chimie analytique de Paris Centre (ED 388), sous la direction de D. Bourgarit et l'encadrement de B. Mille, 251 p. https//tel.archivesouvertes.fr/tel-00918829/, consulté le $1^{\mathrm{er}}$ mai 2017

Bouquillon A., Descamps-Lequime S., Hermary A., Mille B., 2006, « Nouvelle étude sur l'Apollon Chatsworth », Revue Archéologique, 2/2006, p. 227-261.

Daehner J. M., 2015, "Statue of an Athlet (the Croatian Apoxyomenos)", dans Exp. Florence-Los Angeles, 2015, $\mathrm{n}^{\circ} 41$, p. $274-275$

Exp. Florence-Los Angeles-Washington, 2015 : Power and Pathos. Bronze Sculpture of the Hellenistic World [Exposition, Florence, Palazzo Strozzi, 14 March-21 June 2015 ; Los Angeles, J. Paul Getty Museum, 28 July-1 November 2015 Washington D.C., National Gallery of Art, 6 December 2015-20 March 2016, Daehner J. M., Lapatin K. (eds.)], Giunti Editore, Fondazione Palazzo Strozzi, J. Paul Getty Trust.

Formigli E., 1984 : « La tecnica di costruzione delle statue di Riace ", dans Vlad Borelli L. et Pelagatti P. (eds), Due Bronzi da Riace. Rinvenimento, restauro, analisi ed ipotesi di interpretazione, 1-2 (Bollettino d'Arte 3, serie speciale) Roma, p. 107-142.

Formigli E., 2012, « La datazione tecnologica dei grandi bronzi antichi: il caso della Lupa Capitolina », dans M. Denoyelle, S. Descamps, B. Mille, S. Verger (eds.), Bronzes Grecs et Romains, recherches récentes, Hommage à Claude Rolley, 16-17 juin 2009, INHA, http://inha.revues.org/3956 (consulté le 25 mai 2017).

Hemingway S., 2004, The Horse and Jockey

from Artemision. A Bronze Equestrian Monument of the Hellenistic Period,
University of California Press, Berkeley, Los Angeles, London.

Karniš Vidovič I., Mille B., 2015, "The Croatian Apoxyomenos - New Insights into its Manufacturing Technique", dans New Research on Ancient Bronzes. Acta of the XVIIIth International Congress on Ancient Bronzes (Zurich, September 3-7, 2013), Deschler-Erb E. et Della Casa P. (eds.), Zurich Studies in Archaeology, Vol. 10, 2015, p. 97-105.

Karniš Vidovič I., Mille B., à paraître, soumis en 2016, "New Results on the Alloys of the Croatian Apoxyomenos", dans Lapatin, K., Daehner, J. (eds.), 19th International Congress on Ancient Bronzes, 13-17 octobre 2015, Los Angeles, Getty Trust.

Mattusch C.C., 1996, The Fire of Hephaistos. Large Classical Bronzes from North American Collection, Harvard University Art Museum, Cambridge, Massachusetts

Mille B., 2012, "The Casting Techniques of Antique South Arabian Large Bronze Statues", dans P. Jett, B. McCarthy, J. G. Douglas (eds.), Scientific Research on Ancient Asian Metallurgy: Proceedings of Fifth Forbes Symposium at the Freer Gallery of Art, Washington, Smithsonian Institution, 28-29/10/2010, Archetype Publications Ltd, p. 225-247.

Mille B., Rossetti L., Rolley C., Bourgarit D., Formigli E., Pernot M., 2012, « Les deux statues d'enfant en bronze (Cap d'Agde) : étude iconographique et technique ", dans M. Denoyelle, S. Descamps-Lequime, B. Mille et S. Verger (eds.), Bronzes Grecs et Romains, recherches récentes, Hommage à Claude Rolley, 16-17 juin 2009, INHA, http://inha.revues.org/3949 (consulté le 25 mai 2017)

Mille B., Descamps-Lequime S., à paraître, soumis en 2016, "A Technological Re-Examination of the Piombino Apollo", dans Lapatin K., Daehner J. (eds.), 19th International Congress on Ancient Bronzes, 13-17 octobre 2015, Los Angeles, Getty Trust.

Schalles H.-J., Pelz U., 2011, Der Xantener Knabe. Technologie, Ikonographie, Funktion und Datierung, (Xantener Berichte 22), Philip von Zabern, Darmstadt 